\title{
Comunicação
}

\section{Diagnóstico de hipertensão arterial sistêmica: evidências de que os critérios contemporâneos devem ser revistos}

\author{
F.D. Fuchs, J. Lubianca Neto, R.S. Moraes, J.C. Jotz, L. Wannmacher, G.A. Rosito, C.L. de Paoli, \\ L.B. MOREIRA
}

Unidade de Farmacologia Clínica do Hospital de Clínicas de Porto Alegre e Departamento de Farmacologia, Universidade Federal do Rio Grande do Sul, Porto Alegre, RS.

\begin{abstract}
RESUMO - Diversos comitês normativos em hipertensão arterial recomendam considerar a média de várias aferições da pressão arterial com esfigmomanômetro para diagnosticar hipertensão. Não há consenso sobre o número de medidas a serem consideradas.

Овј етіvo. Descrever o comportamento da pressão arterial obtida em três dias diferentes, utilizando-se a média de seis aferições para o diagnóstico de hipertensão.

Métodos. No ambulatório de hipertensão da Unidade de Farmacologia Clínica do Hospital de Clínicas de Porto Alegre, emprega-se a média de seis determinações, obtidas em três dias diferentes, para diagnosticar e classificar a hipertensão, exceto nos pacientes com valores muito baixos ou elevados nas duas primeiras aferições. Cinqüenta e oito pacientes foram submetidos a essa rotina.

Resultados. As médias das pressões sistólica (PS) e diastólica (PD) decresceram da primeira à sexta determinação (ANOVA para medidas repeti-
\end{abstract}

\section{INTRODUÇÃO}

A hipertensão arterial sistêmica (HAS), em decorrência direta de seus efeitos sobre múltiplos sistemas ou como fator de risco para aterosclerose, é uma importante causa de morbimortalidade da população adulta brasileira ${ }^{1}$. A detecção e o tratamento dos hipertensos severos e moderados reduz a morbimortalidade de forma importante ${ }^{2,3}$. Mais recentemente, demonstrou-se que também os hipertensos leves, incluindo os idosos, se beneficiam do tratamento ${ }^{4,5}$. Não há consenso entre diversos comitês normativos internacionais e naci onais sobre os valores de pressão arterial sistólica e diastól i ca que indicam necessi dade de intervenção terapêutica ${ }^{6-11}$.

O diagnóstico errôneo pode ter implicações emocionais $^{12}$ e físicas, pois a redução acentuada da pressão diastólica em indivíduos com cardiopatia isquêmi ca subclínica talvez possa aumentar a incidência de eventos coronarianos ${ }^{13-16}$. das: $F=4,45, p=0,001$ para $P S$ e $F=5,54, p<0,001$ para PD). Os pacientes foram divididos em grupos com PS e PD obtidas na primeira aferição superiores e inferiores à média de todo grupo. A di minuição de ambas as pressões ao longo das seis aferições ficou restrita aos grupos com valores da primeira determinação superiores à média de todo o grupo (ANOVA: $F=8.03 ; p<0,0001$ para PS $e$ $F=6,33, p<0,0001$ para $P D)$. A regressão à média $e$ uma reação de alerta inicial são explicações aventadas para esse fenômeno.

ConClusão. E sses dados demonstram que o diagnóstico de hipertensão arterial não deve ser feito com base em uma única aferição e sugerem que a recomendação de diagnosticar hipertensão severa baseando-se em altos valores das duas primeiras medidas pode classificar erroneamente alguns pacientes.

UNITERMOS. Hipertensão. Pressão arterial. Diagnóstico. Medidas repetidas.

Assim, é crítico classificar corretamente a pressão arterial dos indivíduos para não tratar aquel es que não se beneficiarão. O registro intraarterial pode ser considerado como padrão ouro. A monitorização ambulatorial da pressão arterial (MAPA) e a autodeterminação registram valores mais próximos do padrão ouro ${ }^{17}$, mas não são factíveis em bases populacionais. As medidas casuais por esfigmomanometria costumam ser mais el evadas, especialmente se o aferidor for um médico ${ }^{18-20}$.

Quanto às técnicas de aferição da pressão arterial, todos os comitês recomendam, de forma vaga, repetir as medidas várias vezes antes de diagnosti car um paciente como hi pertenso, mas não especificam quais medi das devem ser consi deradas. $\mathrm{Na}$ prática diária, freqüentemente, estabelece-se o diagnóstico somente com os valores el evados em uma ou duas aferições.

No ambulatório de hipertensão arterial do Programa de Farmacologia Clínica do Hospital de 
Clínicas de Porto Alegre desenvol ve-se um estudo de coorte de pacientes hipertensos, prospectivamente planejado ${ }^{21}$. Analisando-se os dados obtidos na avaliação inicial dos pacientes, verificou-se que os critérios diagnósticos contemporâneos de hipertensão arterial não são satisfatórios, como se pode observar na presente comunicação.

\section{PACIENTES E MÉTODOS}

A maioria dos pacientes chega ao ambulatório com o diagnóstico prévio de hipertensão. Mesmo assim, são submeti dos a uma avaliação sistematizada, de acordo com as recomendações internacionais e brasileiras ${ }^{22}$. A pressão arterial é determinada com o paciente sentado, após cinco minutos de repouso, através do método auscultatório indireto, com esfigmomanômetros de mercúrio ou aneróides, estando o braço do paciente apoiado e na altura do precórdio. Os primeiros são periodicamente calibrados contra um aparelho padrão de coluna de mercúrio. A primeira fase dos sons de Korotkoff é considerada como pressão sistólica e a quinta, como diastólica. As duas primeiras aferições são feitas em cada braço e as subseqüentes no direito, a não ser que o valor à esquerda seja, no mínimo, $5 \mathrm{~mm}$ de $\mathrm{H} \mathrm{g}$ mais el evado do que à direita. O manguito utilizado é o regular, sendo a pressão obtida corrigida para o diâmetro do braço pela tabela proposta por Maxwell et al. ${ }^{23}$ Todos os profissionais que atendem os pacientes são especificamente treinados na técnica de aferição, sendo avaliados quanto à concordância entre observadores por meio da aferição simultânea da pressão arterial com um estetoscópi o com dupla via de ausculta.

O valor médio da diastólica - calculado com base na primeira e segunda aferições da primeira consulta - estabelece o diagnóstico de hipertensão severa se for superior a $115 \mathrm{~mm}$ de $\mathrm{Hg}$, e de pressão arterial normal quando inferior a $85 \mathrm{~mm}$ de $\mathrm{Hg}$. Os pacientes com valores intermediários são avaliados em duas consultas subseqüentes, separadas por 7 a 10 dias, fazendo-se duas aferições da pressão arterial em cada uma delas. Em cada aferição, respeita-se a rotina especificada anteriormente. Para estes, considera-se a média das seis aferições para diagnosticar hi pertensão e classificar o seu nível. Esta rotina atendeu as recomendações do $4^{\circ}$ relatório do J oint $\mathrm{N}$ ational Committee ${ }^{24}$, vigente na época da coleta dos dados. Todos os passos de uma consulta médica formal são atendidos, com ênfase nos aspectos relacionados à hipertensão. As informações são registradas em arquivo computadorizado para análise de dados e seguimento da coorte.
Tabela 1 - Sexo, idade, peso e altura dos 58 pacientes estudados (média e $\pm \mathrm{EP}$ )

\begin{tabular}{|ccccc|}
\hline Sexo & N & Idade (anos) & Peso (kg) & Altura (cm) \\
Masculino & 18 & $48,2( \pm 2,8)$ & $85,9( \pm 2,9)$ & $169( \pm 1,0)$ \\
Feminino & 40 & $52,6( \pm 2,1)$ & $68,5( \pm 1,6)$ & $154( \pm 1,0)$ \\
\hline
\end{tabular}

Tabela 2 - Pressões arteriais sistólica e diastólica, repetidas em três dias, em 58 pacientes avaliados para diagnosticar hipertensão ( $\mathrm{mmHg}$, média e $\pm \mathrm{EP}$ )

\begin{tabular}{|c|c|c|c|c|c|c|}
\hline \multirow{2}{*}{$\begin{array}{l}\text { Pressão } \\
\text { arterial }\end{array}$} & \multicolumn{6}{|c|}{ Medições } \\
\hline & $1 \underline{a}$ & $2^{\underline{a}}$ & 3 a & $4^{a}$ & $5^{a}$ & $6 \mathrm{a}^{\mathrm{a}}$ \\
\hline Sistólica* & $\begin{array}{c}159,1 \\
( \pm 3,1)\end{array}$ & $\begin{array}{l}156,2 \\
( \pm 3,0)\end{array}$ & $\begin{array}{r}154,0 \\
( \pm 2,8)\end{array}$ & $\begin{array}{c}152,2 \\
( \pm 3,0)\end{array}$ & $\begin{array}{l}153,5 \\
( \pm 2,9)\end{array}$ & $\begin{array}{r}150,6 \\
( \pm 2,8)\end{array}$ \\
\hline Diastólica** & $\begin{array}{c}96,1 \\
( \pm 1,2)\end{array}$ & $\begin{array}{c}97,1 \\
( \pm 1,3)\end{array}$ & $\begin{array}{c}97,0 \\
( \pm 1,5)\end{array}$ & $\begin{array}{c}96,2 \\
( \pm 1,5)\end{array}$ & $\begin{array}{c}94,0 \\
( \pm 1,5)\end{array}$ & $\begin{array}{r}92,7 \\
( \pm 1,5)\end{array}$ \\
\hline
\end{tabular}

*ANOVA: $F=4,45, p=0,001 ; \quad$ ** ANOVA: $F=5,54, p<0,001$.

$\mathrm{Na}$ presente análise, observou-se o comportamento da pressão arterial em 58 pacientes que não estavam usando anti-hipertensivos, determinada em seis ocasiões. Primeiramente, analisou-se o grupo como um todo. Após, constituíram-se dois subgrupos, caracterizados por terem o valor da primeira aferição mai or ou menor do que a média de toda a amostra. A análise estatística foi feita por meio de um teste de ANOVA para múltiplas aferições, aplicando-se o teste de contrastes para medidas repetidas.

\section{RESULTADOS}

I dade, sexo e dados antropométricos dos pacientes estudados estão apresentados na tabela 1. A tabela 2 mostra os valores médios de pressão sistólica e diastólica determinados nas seis ocasiões. Tanto a sistólica quanto a diastólica decresceram da primeira para a sexta aferição (ANOVA para medições repetidas: $F=4,45, p=0,001$ para a sistólica, e $F=5,54, p<0,001$ para a diastólica). Os valores mais díspares foram os da sexta aferição em relação às demais, tanto para a sistólica quanto para a diastólica (teste de contrastes).

A tabela 3 apresenta o comportamento das pressões sistólica e diastólica nos subgrupos com a primeira medida acima e abaixo da média de toda a amostra. O decréscimo da pressão arterial ficou restrito aos pacientes com pressão arterial mais el evada na primeira aferição (ANOVA: $F=8,03, p<$ 0,0001 para a sistólica, e $F=6,33, p<0,0001$ para a 


\begin{tabular}{|c|c|c|c|c|c|c|c|c|}
\hline \multicolumn{2}{|c|}{ Pressão arterial } & \multirow[t]{2}{*}{$\mathbf{N}$} & \multicolumn{6}{|c|}{ Medições } \\
\hline & & & 1 & 2 & 3 & 4 & 5 & 6 \\
\hline \multirow{4}{*}{ Sistólica } & $\geq 158$ (a) & 29 & 177,6 & 174,0 & 167,7 & 165,8 & 165,2 & 161,6 \\
\hline & & & $( \pm 3,6)$ & $( \pm 3,5)$ & $( \pm 3,7)$ & $( \pm 3,9)$ & $( \pm 3,7)$ & $( \pm 3,6)$ \\
\hline & $<158$ (b) & 29 & 146,7 & 138,3 & 140,3 & 138,5 & 141,8 & 139,7 \\
\hline & & & $( \pm 1,3)$ & $( \pm 1,6)$ & $( \pm 2,4)$ & $( \pm 2,9)$ & $( \pm 3,1)$ & $( \pm 3,1)$ \\
\hline \multirow{4}{*}{ Diastólica } & $\geq 96$ (c) & 30 & 103,3 & 104,1 & 102,7 & 102,3 & 99,2 & 98,0 \\
\hline & & & $( \pm 1,0)$ & $( \pm 1,2)$ & $( \pm 1,6)$ & $( \pm 1,4)$ & $( \pm 1,4)$ & $( \pm 1,6)$ \\
\hline & ${ }^{3} 96$ (d) & 28 & 88,4 & 89,6 & 90,8 & 89,7 & 88,4 & 87,1 \\
\hline & & & $( \pm 1,2)$ & $( \pm 1,4)$ & $( \pm 2,2)$ & $( \pm 2,2)$ & $( \pm 2,2)$ & $( \pm 2,2)$ \\
\hline
\end{tabular}

(a): $F=8,03 ; p<0,0001 ;(b): F=0,54 ; p=0,747 ;(c): F=6,33 ; p<0,0001 ;(d): F=1,30 ; p=0,296$

diastólica). A ANOVA para os grupos com pressão inicial inferior à média não demonstrou variações estatisticamente significantes $(F=0,54, p=0,747$ para a sistólica, e $F=1,30, p=0,269$ para a diastólica). A figura ilustra o fenômeno observado.

\section{DISCUSSÃO}

Esses resultados demonstram importante redução da pressão arterial ante a aferi ção repetida em consultório médico. Pelo menos parte desse comportamento pode ser atribuído à regressão à média ${ }^{25}$. A síndrome do "avental branco", diagnosticada pela diferença entre os valores de pressão arterial obtidos em repetidas aferições em consultório com aqueles registrados pela MAPA ${ }^{26,27}$, não expl icaria o comportamento da pressão arterial em nossa observação. A hiper-reatividade de alguns pacientes nas primeiras aferições pode, entretanto, ter ocorrido, consistindo em uma explicação adicional para o fenômeno observado.

Os estudos de coorte que identificaram a elevação de pressão arterial como fator de risco para doença cardiovascular utilizaram a primeira, a segunda ou a média das duas únicas aferições ${ }^{28}$. Por outro lado, a maioria dos ensaios clíni cos que investigaram os efeitos dos anti-hipertensivos sobre a morbimortalidade empregaram a média de duas entre a quinta e sétima medidas ${ }^{29-31}$, para excluir os pacientes com tendência a queda da pressão arterial com a repetição das aferi ções. E sse fato sugere até mesmo que o verdadeiro risco da hipertensão seja maior do que o identificado nos estudos de coorte, pois muitos dos indivíduos com pressão arterial momentaneamente elevada não

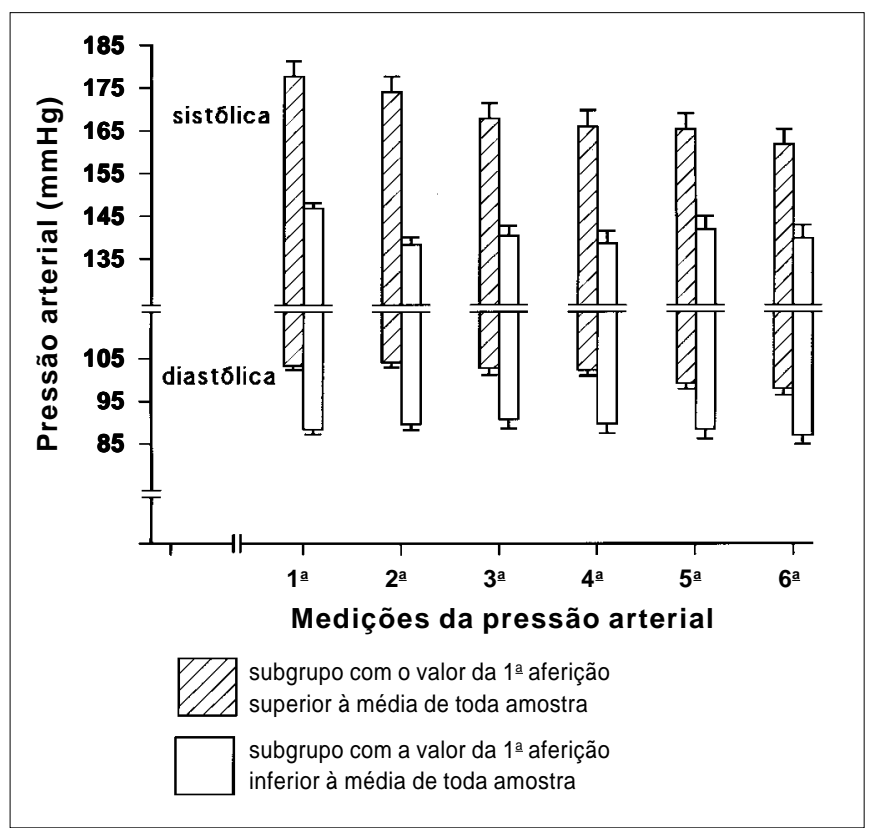

Fig. - Pressões sistólica e diastólica nos pacientes classificados de acordo com o valor da 1a aferição.

seriam hipertensos de fato ${ }^{32}$. Assim, mesmo que o diagnóstico de hipertensão se baseie em medidas de somente um dia, não estará distante da metodologia dos estudos que estabel eceram o risco decorrente da elevação da pressão arterial.

Demonstrou-se que a MAPA é mais poderosa em discriminar o prognóstico dos pacientes hipertensos ${ }^{33,34}$. Provavelmente, isso se deve ao fato de $20 \%$ a $30 \%$ dos indivíduos classificados como hipertensos pela aferição casual, mesmo que repetida, serem normotensos ${ }^{27,35}$.

O J oint National Committee e outros recomen- 
dam que altos valores observados no primeiro dia são diagnósticos ${ }^{6,36}$. Os dados apresentados mostram que esse critério pode classificar erroneamente mais pacientes do que a média de seis medidas, pois o decréscimo da pressão é mais acentuado naqueles com altos valores no primeiro dia. Mesmo a decisão de empregar a média de seis aferições para os valores "leves e moderados" no primeiro dia não reproduz os critérios empregados em ensai os clínicos, permanecendo "contaminada" pel os val ores espúrios do primeiro dia. A repercussão individual de uma classificação errônea é evidente. Dependendo do número de aferições e dos valores observados, um indivíduo pode ser considerado predisposto à hi pertensão (pressão arterial normal-al ta) ou já um hipertenso. Para a primeira situação, recomendar-se-iam medidas de prevenção primária da doença, envolvidas pelo clima positivo que acompanha abordagens preventivas. Se o diagnóstico fosse de hipertensão, no entanto, as repercussões negativas provocadas pelo estigma de doente seriam mais provávei $\mathrm{s}^{12}$, mesmo sendo as medidas terapêuticas iniciais similares às de prevenção primária.

Vários investigadores têm abordado o problema da variabilidade da pressão arterial e sua implicação para o diagnóstico de hipertensão ${ }^{37-40}$, demonstrando o decréscimo dos valores pressóri cos ante medições repetidas. Há evidências de que a sistólica tende a estabilizar entre a 2a e 3a consultas e a diastólica, entre a primeira e segun$\mathrm{da}^{39}$. E ste fato tem sido só parcialmente considerado para o estabelecimento dos critérios diagnósticos contemporâneos, claramente controversos e não consensuais ${ }^{6-11}$.

Em síntese, os dados da presente observação, obtidos de acordo com recomendações padronizadas, demonstram que hipertensão arterial ainda é uma doença comum procurando critérios diagnósticos consensuais e pragmáticos. Enquanto estes inexistirem, parece recomendável não rotular como doentes indivíduos assintomáticos com valores elevados em um único dia (mesmo com várias aferições) e, muito menos, instituir medidas terapêuticas neste momento. Talvez a média da quinta e sexta aferições, obtidas somente na terceira consulta, guarde mel hor correl ação com o prognóstico e a M APA, uma hipótese em investigação em nossa coorte.

Trabal ho financiado, em parte, pelo CNPq (№ 820514/ 89.6) e PROPESP — UFRGS.

\section{SUMMARY}

Hypertension diagnoses: some evidences suggesting criteria changes
It has been recommended to take the average of several blood pressure (BP) determinations with a sphygmomanometer to diagnose hypertension, but there is no agreement on the reading numbers.

PURPOSE. Describing the behavior of BP readings taken in three different days to establish the classificatory BP in an outpatient hypertension clinic.

METHOD. In the outpatient hypertension clinic of the Clinical Pharmacology Division of the Hospital de Clínicas de Porto Alegre, we use the mean of six readings taken in three different days to establish the classificatory blood pressure, except for those with very high or low values in the first day. In this report we describe the behavior of BP in 58 patients submitted to this routine.

Results. The mean of systolic (SBP) and diastolic (DBP) blood pressures decreased from the first to the 6th reading (ANOVA for repeated measurements: $F=4.45, P=0.001$ for $\mathrm{SBP}$ and $\mathrm{F}$ $=5.54, \mathrm{P}<0.001$ for DBP). Afterward, the patients were classified into two groups according their first SBP and DBP reading. The decreasing in both SBP and DBP was confined to those with the first measurement in the upper half of the entire group (ANOVA: $F=8.03 ; P<0.0001$ for $\mathrm{SBP}$ and $\mathrm{F}=6.33$, $P<0.0001$ for DBP). Regression to the mean and some reactiveness in the first day are possible explanations for this.

Conclusion. These data corroborate that the hypertension diagnosis should not be based in an single blood pressure determination and suggest that the recommendati on to diagnose severe hyper tension based on high values in the first two readings could misclassify some patients. [Rev Ass Med Brasil 1997; 43(3): 223-7.]

KEY WORDS: Hypertension. Blood pressure. Several readings.

\section{REFERÊNCIAS BIBLIOGRÁFICAS}

1. Ministério da Saúde. Normas técnicas para o Programa Nacional de Educação e Controle da Hipertensão Arterial (PNECHA). Brasília, Centro de Documentação do Ministério da Saúde, 1988.

2. Veterans Administration Cooperative Study Group on An Antihypertensive Agents. Effects of treatment on morbidity in hypertension. Results in patients with diastolic blood pressures averaging 115 through $129 \mathrm{mmHg}$. J AMA 1967; 202: 116-22.

3. Veterans Administration Cooperative Study Group on Antihypertensive Agents. Effects of treatment on morbidity in hypertension. II. Results in patients with diastolic blood pressures averaging 90 through $114 \mathrm{mmH}$. J AMA 1970; 213: 1.143-52.

4. Collins R, Peto R, MacMahon S et al. Blood pressure, stroke, and coronary heart disease. Part 2, short-term reductions in 
blood pressure: overview of randomised drug trials in their epidemiological context. Lancet 1990; 335: 827-38.

5. Beard K, Bulpitt C, Mascie-Taylor $\mathrm{H}$ et al. Management of elderly hypertensive with sustained hypertension. $\mathrm{Br}$ Med J 1992; 304: 412-6.

6. J oint National Committee. The fifth report of the J oint National Committee on detection, evaluation, and treatment of high blood pressure (J NC V). Arch Intern Med 1993; 153: 154-83.

7. The Guidelines Subcommittee of the WHO/ISH Mild Hypertension Liaison Committee. 1993 guidelines for the management of mild hypertension. Hypertension 1993; 22: 392-403.

8. Sever $P$, Beevers $G, B$ ulbitt $C$ et al. Management guidelines in essential hypertension: report of the second working party of the British Hypertension Society. Br Med J 1993; 306: 983-7.

9. Haynes RB, Lacourcière $Y$, Rabkin SW et al. Report of the Canadian Hypertension Society Consensus Conference: 2. Diagnosis of hypertension in adults. Can Med Assoc J 1993; 149: 409-18.

10. J ackson R, Barham P, Bills J et al. Management of raised blood pressure in New Zealand: a discussion document. $\mathrm{Br}$ Med J 1993; 307: 107-10.

11. II Consenso Brasileiro de Hipertensão Arterial. Rev Bras Nefrol 1994; 16(supl 2): S260-S278.

12. Bloom J R, Monterossa S. Hypertension labeling and sense of well-being. Am J Public Health 1981; 71: 1.228-32.

13. Cruickshank J M, Thorp J M, Zacharias FJ. Benefits and potential harm of lowering high blood pressure. Lancet 1987; 1: $581-4$

14. Farnett $L$, Mulrow CD, Linn WD, Lucey CR, Tuley MR. The J curve phenomenon and the treatment of hypertension. J AMA 1991; 265: 489-95.

15. McCloskey LW, Psaty BM, Koepsell TD, Aagaard GN. Level of blood pressure and risk of myocardial infarction among treated hypertensive patients. Arch Intern Med 1992; 152: 513-20.

16. D'Agostino RB, Belanger AJ, Kannel WB, Cruickshank J M. Relation of low blood pressure to coronary heart disease death in the presence of myocardial infarction: the Framingham study. Br Med J 1991; 303: 385-9.

17. Graettinger WF, Lipson J L, Cheung DG, Weber MA. Validation of portable noninvasive blood pressure monitoring devices: comparisons with intra-arterial and sphygmomanometer measurements. Am Heart J 1988; 116: 1.155-60.

18. White WB, Morganroth J. Usefulness of ambulatory monitoring of blood pressure in assessing antihypertensivetherapy. Am J Cardiol 1989; 63: 94-8.

19. Zachariah PK, Sheps SG, Smith RL. Clinical use of home and ambulatory blood pressure monitoring. Mayo Clin Proc 1989; 64: 1.436-46.

20. Marolf AP, Hany S, Battig B, Wetter W. Comparison of casual ambulatory and self-determined blood pressure measurements. Nephron 1987; 47 (suppl I): 142-5.

21. Fuchs FD, Wannmacher $L$, Moraes $R$ et al. Um projeto de atendimento ambulatorial e seguimento de pacientes hipertensos: objetivos, funcionamento e primeiros resultados. Rev Hosp Clín Porto Alegre 1989; 9: 161-4.
22. Fuchs FD. Hipertensão arterial sistêmica: avaliação e tratamento. In Duncan BB, Schmidt MI, Giugliane ER (eds): Medicina ambulatorial: condutas clínicas em atenção primária. Porto Alegre, Artes Médicas, 1990; 302-25.

23. Maxwell MH, Schroth PC, Waks AU, Karam M, Dornfeld LP. Error in blood pressure measurement due to incorrect size in obese patients. Lancet 1982; 2: 33-6.

24. J oint National Committee. The 1988 report of the J oint National Committee on detection, evaluation, and treatment of high blood pressure. Arch Intern Med 1988; 148: 1.023-38.

25. Fletcher $\mathrm{RH}$, Fletcher SW, Wagner $\mathrm{EH}$. Clinical epidemi ol ogy - the essentials. Baltimore, Williams \& Wilkins, 1982, 37-9.

26. Mancia G, Bertinieri G, Grassi G et al. Effects of blood pressure measurement by the doctor on patient's blood pressure and heart rate. Lancet 1983; 2: 695-8.

27. Pickering TG, J ames GD, Boddie $C$ et al. How common is white coat hypertension? J AMA 1988; 259: 225-8.

28. Pooling Project Cooperative Group: relationship of blood pressure, serum cholesterol, smoking habit, relative weight and ECG abnormalities to incidence of major coronary events: final report of the National Cooperative Pooling Project. J Chron Dis 1978; 31: 201-306.

29. Hypertension Detection and Follow-up Program Cooperative Group. Five-year findings on the Hypertension Detection and Follow-up Program: I. Reduction in mortality of persons with high blood pressure, including mild hypertension. J AMA 1979; 242: 2.562-71.

30. Medical Research Council Working Party. MRC trial of treatment of mild hypertension: principal results. $\mathrm{Br} \mathrm{Med} J$ 1985; 291: 97-104.

31. Multiple Risk Factor Intervention Trial Research Group. Multiple risk factor intervention trial: risk factors changes and mortality results. J AMA 1982; 248: 1465-77.

32. 1. MacMahon S, Peto R, Cutler J et al. Blood pressure, stroke, and coronary heart disease. Part 1, prolonged differences in blood pressure: prospective observational studies corrected for the regression dilution bias. Lancet 1990; 335: 765-74.

33. Pickering TG, Devereux RB. Ambulatory monitoring of blood pressure as a predictor of cardiovascular risk. Am Heart J 1987; 114: 925-8.

34. Perloff $D$, Sokolow M, Cowan R. The prognostic value of ambulatory blood pressures. J AMA 1983; 249: 2.792-8.

35. Weber MA. Automate blood pressure monitoring: a new dimension in diagnosis [Editorial]. Mayo Clin Proc 1988; 63: 1.151-3.

36. Rosner B, Polk BF. The implications of blood pressure variability for clinical and screening purposes. J Chron Dis 1979; 32: 451-61.

37. Armitage $P, F$ ox $W$, Rose GA, Tinker CM. The variability of measurements of casual blood pressure. Clin Sci 1966; 30: 337-44.

38. Rosner B, Polk BF. The instability of blood pressure variability over time. J Chron Dis 1981; 34: 135-9.

39. Hartley RM, Velez R, Morris RW, D'Souza MF, Heller RF. Confirming the diagnosis of mild hypertension. $\mathrm{Br}$ Med J 1983; 286: 287-9.

40. Ambrosio GB, Dowd J E, Strasser T, Tuomilehto J. The dynamics of blood pressure in populations and hypertensive cohorts. Bull World Health Org 1986; 64: 93-9. 